Published in final edited form as:

Clin Gastroenterol Hepatol. 2014 February ; 12(2): 265-73.e1. doi:10.1016/j.cgh.2013.03.034.

\title{
Risk of Cancer in Patients with Inflammatory Bowel Diseases: a Nationwide Population-Based Cohort Study with 30 Years of Follow Up
}

\author{
Michael D. Kappelman ${ }^{1}$, Dora K. Farkas ${ }^{2}$, Millie D. Long ${ }^{1}$, Rune Erichsen², Robert S. \\ Sandler ${ }^{1}$, Henrik T. Sørensen ${ }^{2}$, and John A. Baron ${ }^{1}$ \\ ${ }^{1}$ University of North Carolina at Chapel Hill, Chapel Hill, NC, United States \\ ${ }^{2}$ Aarhus University Hospital, Aarhus, Denmark
}

\begin{abstract}
Background \& Aims-Data regarding the risk of gastrointestinal and extra-intestinal cancers in Crohn's disease (CD) and ulcerative colitis (UC) are needed to understand the clinical course of inflammatory bowel diseases (IBDs) and their treatments.
\end{abstract}

\begin{abstract}
Methods-We performed a nationwide historical cohort study using Danish healthcare databases. We identified patients with a diagnosis of CD or UC, recorded from 1978 through 2010, and followed them until first occurrence of cancer, death, or emigration. We used standardized incidence ratios (SIRs) to compare cancer incidence in CD and UC patients to that expected in the general population.

Results-Excluding cancers diagnosed within 1 year of IBD diagnosis, 772 cases of invasive cancer occurred among 13,756 patients with CD (SIR, 1.3; 95\% confidence interval [CI], 1.2-1.4) and 2331 occurred among 35,152 patients with UC (SIR, 1.1; 95\% CI, 1.0-1.1). CD was weakly associated with gastrointestinal cancers (SIR 1.2; 95\% CI, 1.0-1.4) and extra-intestinal cancers
\end{abstract}

(C) 2013 The American Gastroenterological Association. Published by Elsevier Inc. All rights reserved.

Information for correspondence: Michael Kappelman, MD, MPH, University of North Carolina Chapel Hill, Department of Pediatrics, Division of Pediatric Gastroenterology, 130 Mason Farm Road, campus box 7229, Chapel Hill, NC 27599, Phone: (919) 966-1343, Fax: (919) 966-8641, michael_kappelman@med.unc.edu.

Publisher's Disclaimer: This is a PDF file of an unedited manuscript that has been accepted for publication. As a service to our customers we are providing this early version of the manuscript. The manuscript will undergo copyediting, typesetting, and review of the resulting proof before it is published in its final citable form. Please note that during the production process errors may be discovered which could affect the content, and all legal disclaimers that apply to the journal pertain.

Disclosures: No authors have a conflict of interest to disclose

Author Contributions:

MDK: study concept and design; analysis and interpretation of data; drafting of the manuscript; critical revision of the manuscript for important intellectual content.

DFK: study concept and design; acquisition of data; analysis and interpretation of data; critical revision of the manuscript for important intellectual content; statistical analysis.

MDL: drafting of the manuscript; analysis and interpretation of data; critical revision of the manuscript for important intellectual content.

RE: analysis and interpretation of data; critical revision of the manuscript for important intellectual content.

RSS: analysis and interpretation of data; critical revision of the manuscript for important intellectual content.

HTS: study concept and design; acquisition of data; analysis and interpretation of data; critical revision of the manuscript for important intellectual content; obtained funding; study supervision.

JAB: study concept and design; analysis and interpretation of data; critical revision of the manuscript for important intellectual content; study supervision. 
(SIR, 1.3; 95\% CI, 1.2-1.4), with the strongest associations for hematological malignancies (SIR, 1.9; 95\% CI, 1.5-2.3), smoking-related cancers (SIR 1.5, 95\% CI 1.3-1.8), and melanoma (SIR, $1.4 ; 95 \% \mathrm{CI}, 1.0-1.9)$. Associations between UC and gastrointestinal and extra-intestinal cancers were weaker (SIR, 1.1; 95\% CI, 1.0-1.2 and SIR, 1.1; 95\% CI, 1.0-1.1, respectively). The relative risk of extra-intestinal cancers among patients with IBD was relatively stable over time, although the risk of gastrointestinal cancers decreased.

Conclusions-Patients with IBD, particularly $\mathrm{CD}$, are at increased risk for gastrointestinal and extra-intestinal malignancies. The relative risk of gastrointestinal malignancy has deceased since 1978, without a concomitant increase in the risk of non-gastrointestinal malignancy.

\section{Keywords}

Epidemiology; Crohn's disease; ulcerative colitis; colorectal cancer; GI tumor

\section{INTRODUCTION}

Gastrointestinal cancers are widely acknowledged as a long-term complication of inflammatory bowel diseases, likely as a result of chronic inflammation. Therefore, it is possible the medical therapy that decreases intestinal inflammation might protect against gastrointestinal malignancy. A recently published population based study in Denmark demonstrated a decreasing risk of colorectal cancer over the past 3 decades, providing some support for this hypothesis. ${ }^{1}$ However, a North American study yielded conflicting results, demonstrating a stable risk over time, despite increasing use of immunosuppressive and biological therapies. ${ }^{2}$

In addition to gastrointestinal malignancy, patients with IBD may also be at increased risk of extra-intestinal malignancy, including lymphoma ${ }^{3-5}$, non-melanoma skin cancer (NMSC) $^{6-9}$, and melanoma ${ }^{9}$ among others. A meta-analysis of eight population-based cohort studies showed a small increase in the risk of selected extra-intestinal malignancies in IBD patients (SIR, 1.10; 95\% confidence interval (CI) 0.96-1.27). However, most of the studies included data collected prior to the widespread use of immunosuppressive agents and biologics ${ }^{10}$, whose use may affect cancer risk.

If immunosuppressive medications reduce the risk of gastrointestinal malignancy by suppressing intestinal inflammation but increase the risk of extra-intestinal malignancies, IBD patients may be trading off one set of risks for another. We therefore sought to comprehensively evaluate both intestinal and extra-intestinal malignancies, as well as the occurrence of any invasive cancer, in a nationwide cohort of patients with IBD in Denmark. We also sought to evaluate whether the IBD-associated risks have changed over the last three decades.

\section{METHODS}

We performed a nationwide, historical cohort study in Denmark to compare the number of cancers occurring in patients with a registered diagnosis of $\mathrm{CD}$ and $\mathrm{UC}$ to the number of 
cancers expected in the general population, after standardization by sex, age, and calendar period (1 year intervals).

\section{Data Sources}

The Danish Civil Registration System (DCRS) assigns a personal identifier to each Danish resident at the time of birth or immigration, and also monitors the occurrence of death or emigration from the country. ${ }^{11}$ The DCRS thus can provide denominator data for event rates in the population. The civil registration number can link individuals throughout the numerous registries maintained in Denmark.

The Danish National Patient Register (DNPR) contains records on all acute care hospital discharges occurring after January 1, 1977. ${ }^{12}$ Since 1995, the database has also included hospital outpatient visits, accounting for essentially all specialist gastroenterology and cancer care in Denmark. Data elements include civil registration number, dates of hospital admission and discharge, surgical procedures, and up to 20 discharge diagnoses for each hospitalization. All inpatient and outpatient hospital visits are assigned diagnosis codes according to the International Classification of Diseases $\left[8^{\text {th }}\right.$ revision, (ICD-8) until the end of 1993 and $10^{\text {th }}$ revision (ICD-10) thereafter].

The Danish Cancer Registry (DCR) has prospectively recorded all cases of incident cancers diagnosed in Denmark since 1943. ${ }^{13}$ Cancers diagnosed after January 1, 2004 are classified according to ICD-10. Cancers diagnosed previously have been re-coded in this format. Comprehensive evaluation has shown that the Registry is 95 to 98 percent complete and valid. ${ }^{13}$

\section{Study population and IBD exposure classification}

The source population consisted of all Danish residents during the time period spanning January 1, 1978 through December 31, 2010 ( $\mathrm{n}=7,955,034 ; 171.4$ million person-years of follow up). We used the DNPR to identify patients with Crohn's disease (CD) and ulcerative colitis (UC). We considered patients with ICD-8 code 563.01 and/or ICD-10 K50 to have $\mathrm{CD}$, and patients with ICD-8 code 563.19 and/or ICD-8 code 569.04, and/or ICD-10 K51 to have UC. To avoid diagnostic ambiguity, patients with codes for both conditions were excluded from all analyses. Prior work has validated the completeness and accuracy of the DNPR for identifying patients with $\mathrm{IBD}^{14}$ and other chronic illnesses ${ }^{15}$. For IBD, a validation study compared diagnoses of $\mathrm{CD}$ and $\mathrm{UC}$ recorded in the database with hospital and pathology records and found the sensitivity to be $94 \%$ for both CD and UC, and the specificity to be $97 \%$ for CD and $90 \%$ for UC respectively. ${ }^{14}$

\section{Cancer outcome classification}

The following cancer outcomes were identified from the DCR: 1) any invasive malignancy, excluding NMSC, 2) gastrointestinal cancers (colorectal, small intestine, and other gastrointestinal cancers including those in the esophagus, stomach, liver, gallbladder and biliary tract, pancreas, and anal canal), 3) invasive extra-intestinal cancers, and 4) NMSC. We also analyzed the following extra-intestinal cancer groupings: a) hematological malignancies (Hodgkin's lymphoma, non-Hodgkin's lymphoma, lymphoid leukemia, 
myeloid leukemia, and monocytic leukemia) because of their prior associations with immunosuppressive medication use $\mathrm{e}^{3-5}$; b) female reproductive cancers (breast, ovary, and uterus) because emerging data suggests that female sexual hormones, including oral contraceptives and hormone replacement therapies, may increase the risk of IBD itself ${ }^{16,17}$; and c) smoking-related cancers (lung, larynx, oral cavity \& pharynx, bladder, kidney, and ureter) because of the well described associations between tobacco use and IBD risk. ${ }^{18}$ Additionally, we separately analyzed selected other common malignancies including prostate, lung, bladder, melanoma, breast, and uterus ${ }^{19}$.

Cancers occurring within one year of a registered diagnosis of IBD may be due to a number of factors including: 1) First recording of prevalent IBD in the DNPR at the time health care was sought for cancer, 2) Increased detection of cancer following IBD diagnosis due to increased health care contact/detection, 3) Co-diagnosis of IBD and cancer simultaneously due to overlapping symptoms and diagnostic procedures, and 4) Misclassification of IBD or cancer. Therefore, our main analyses focus on cancers occurring one or more years following the date that the diagnosis of CD or UC was first recorded in the Patient Registry, consistent with the approach taken by others. ${ }^{1}$

\section{Analysis}

We constructed cohorts of CD and UC patients who had not had a recorded diagnosis of invasive cancer prior to their first registered diagnosis of CD or UC. Each patient was followed from the first recorded IBD diagnosis until first occurrence of invasive cancer, death, emigration, or end of the study period. For cancer types occurring in $\geq 5$ patients, standardized incidence ratios (SIRs) were used to compare the observed number of cancers to the expected number, calculated on the basis of national incidence rates obtained from the DCR according to sex, age, and calendar period in one-year intervals. Multiplying the number of person-years of observation by the incidence rates yielded the number of cancer cases that would be expected if patients with IBD had the same risk of cancer as the general population.

Confidence intervals were computed based on the assumption that the observed number of cases follows a Poisson distribution. Exact limits were used when the observed number was less than 10; otherwise, Byar's approximation was used. ${ }^{20}$ All analyses were stratified by time from first recorded IBD diagnosis until cancer $(<1$ year or $\geq 1$ year). We also stratified by calendar year of cancer diagnosis (1978-1987, 1988-1997, and 1998-2010), age at IBD diagnosis ( $0-19$ years, $20-39$ years, and $\geq 40$ years), age at cancer diagnosis $(0-29$ years, 30-49 years, 50-69 years, and $\geq 70$ years) and gender. For analyses of colorectal cancer, liver cancer, and gallbladder cancer, SIRs were stratified by the presence of a registered diagnosis of cholangitis (ICD-8 code 57504 and ICD-10 code K83.0) recorded prior to cancer diagnosis. We also performed an additional analysis of colorectal cancers occurring in the most recent time period (1998 to 2010), censoring individuals with UC at the time of colectomy (codes 45060, 45061, 45080, and 45081 from 1978-1995, and codes KJFH thereafter). Finally, in addition to analyzing relative risk, we also calculated the absolute risk of cancers occurring 1-11 years following IBD diagnosis. 
All statistical analyses were performed using SAS version 9.2 (Cary, NC). The study protocol was approved by the Danish Protection Agency (record number 1-16-02-1-08), and granted exemption from review by the Institutional Review Board at the University of North Carolina at Chapel Hill because it involved use of existing, de-identified data.

\section{RESULTS}

\section{Cohort Composition and Overall Cancer Risk}

We identified 13,756 individuals with a registered diagnosis of CD (mean age 40.6 years, median age 36.5 years) and 35,152 individuals with UC (mean age 46.5 years, median age 44.2 years) who were followed for a mean of 7.6 and 7.8 years respectively. We excluded 6,191 individuals with diagnoses for both conditions. Among patients with CD, 208 cases of invasive cancer occurred within the first year of diagnosis (SIR $=3.6,95 \% \mathrm{CI}: 3.1-4.1$ ) and 772 cases in second and subsequent years ( $\mathrm{SIR}=1.3,95 \% \mathrm{CI}$ : 1.2-1.4). Among UC patients, 376 cases of invasive cancer occurred within 1 year ( $\mathrm{SIR}=1.8,95 \% \mathrm{CI}$ : $1.6-2.0$ ) and 2331 cases in second and subsequent years ( $\mathrm{SIR}=1.1,95 \%$ CI: $1.0-1.1)$ (Table 1).

\section{Gastrointestinal Cancers}

After the first year following diagnosis, UC and CD were weakly associated with gastrointestinal cancers overall (SIR 1.1, 95\% CI: 1.0 to 1.2 and SIR 1.2, 95\% CI: $1.0-1.4$ respectively). Associations with specific gastrointestinal cancers are shown in Table 1 and Supplemental Table 1. No associations with colorectal cancer were observed for either CD or UC. A subanalysis of colorectal cancer occurring in UC patients between 1998 and 2010 which censored follow-up at the time of colectomy also showed no excess risk (SIR 1.0, 95\% CI: 0.9-1.2). However, among UC patients with a recorded diagnosis of cholangitis, the risk of colorectal cancer was significantly increased (SIR 8.6, 95\% CI 3.9-16.3). CD was associated with a significantly increased risk of cancers of the small intestine (SIR 8.4, 95\% CI: 4.3-14.7) and gallbladder and biliary tract (SIR 2.4, 95\% CI 1.1-4.5). For UC, there were notable associations for cancers of the liver (SIR 1.6, 95\% CI 1.1-2.2), and gallbladder (SIR 2.5, 95\% CI 1.8-3.5). The SIRs for liver and gallbladder cancer in patients with UC and cholangitis were 80.0 (95\% CI: 32.1-164.8) and 129.1 (95\% CI: 47.4-281.5) respectively, whereas in patients without cholangitis the SIRs were 1.3 (95\% CI 0.9-1.9) and 2.1 (1.4-3.0) respectively.

The absolute risks of selected gastrointestinal cancers are shown in Table 2 and Figure 1. Overall, the absolute risk of gastrointestinal cancers 1-11 years following diagnosis of CD was 1.2 percent (95\% CI: 1.0-1.4). For UC, the overall absolute risk was 1.4 percent (95\% CI 1.2-1.6). Approximately $50 \%$ of gastrointestinal cancers in both CD and UC patients occurred in the colorectum.

\section{Extra-intestinal Cancers}

The risk of extra-intestinal cancers was increased for patients with CD (SIR 1.3, 95\% CI 1.2-1.4), and to a minimal extent for UC (SIR 1.1 (95\% CI 1.0-1.1). In CD, this was largely due to an increased risk of hematological malignancies (SIR 1.9, 95\% CI 1.5-2.3), smokingrelated cancers (SIR 1.5, 95\% CI 1.3-1.8), and melanoma (SIR 1.4, 95\% CI 1.0-1.9). 
Neither CD nor UC were associated with a risk of female reproductive cancers. UC, but not $\mathrm{CD}$, was associated with a statistically significant increase in prostate cancer, though the association was relatively weak (SIR 1.2, 95\% CI 1.1-1.4). In separate analyses, both CD and UC were associated with an increased risk of non-melanoma skin cancer (SIR for CD 2.1, 95\% CI 1.8-2.3 and SIR for UC 1.8, 95\% CI 1.7-2.0).

For both CD and UC, the absolute risks of extra-intestinal cancers, excluding NMSC, were far greater than that of intestinal cancers (Table 2 and Figure 1). For CD, the overall absolute risk of extra-intestinal cancers, excluding NMSC, 1-11 years following diagnosis was 4.4 percent (95\% CI: 4.0-4.9). For UC, the absolute risk was slightly higher at 5.0 percent (95\% CI 4.7-5.3), likely related to the older age of these patients in comparison to those with $\mathrm{CD}$. However, the absolute risk of hematological malignancies was higher among CD patients than UC patients ( 0.7 percent versus 0.4 percent $)$.

\section{Effect of Age of IBD Diagnosis}

For both CD and UC, the relative risk of invasive cancer one or more years after IBD diagnosis was highest for those diagnosed with IBD before 20 years of age (SIR for CD 2.3, 95\% CI 1.5-3.4 and SIR for UC 2.0, 95\% CI 1.4-1.7) (Table 3). This was largely due to the risk of gastrointestinal cancers (SIR for CD 7.3, 95\% CI 2.0-18.6 and SIR for UC 20.2, 95\% CI 13.1-29.8).

\section{Cancers Occurring Early in Life}

The relative risk of cancer occurring early in life, particularly gastrointestinal cancer, was markedly elevated in UC patients (SIR for gastrointestinal cancer diagnosed before 30 years of age 15.8, 95\% CI 7.3-30.1). The relative risk of early-life cancers also appears to be increased in $\mathrm{CD}$, though more modestly (SIR for invasive cancer diagnosed before 30 years of age $1.8,95 \%$ CI 1.1-2.7). More detailed analyses of cancer types occurring early in life were limited by sparse data.

\section{Cancer Relative Risk over Time}

The relative risk of gastrointestinal cancers among patients with CD and UC has decreased over the last 3 decades (Table 3). Between 1978-1987, the SIR of gastrointestinal cancers in patients with CD was 1.9 (95\% CI 1.0-3.4). In the last two decades, the relative risk of gastrointestinal cancers among patients with $\mathrm{CD}$ did not differ from the general population. Similarly the SIR of gastrointestinal cancers in UC patients has decreased from 1.4 during 1979-1987 to 1.1 in the last two decades. Over this same period, the relative risk for extraintestinal cancers and hematological malignancies has remained largely unchanged.

\section{DISCUSSION}

In this population-based nationwide study, we found that patients with $\mathrm{CD}$ and $\mathrm{UC}$ have a $30 \%$ and $10 \%$ higher long term risk of invasive cancer than the general population. The excess risk in $\mathrm{CD}$ patients is largely due to extra-intestinal cancers, particularly hematological malignancies and melanoma, both of which may be related to immune suppression, and smoking-related cancers. With regards to gastrointestinal malignancies, CD 
was associated with a higher risk of cancer of the small intestine, and both CD and UC were associated with an increased risk of hepatobiliary malignancies, particularly in patients with cholangitis.

The absolute risk of invasive malignancy was somewhat higher in the UC cohort, as compared to the $\mathrm{CD}$ cohort, likely related to older age: $6.3 \%$ during the 1-11 years after diagnosis versus $5.6 \%$. The absolute risk of hematological malignancies was nearly twice that in CD patients versus those with UC, though, this risk was lower than that of gastrointestinal malignancies as displayed in Figure 1.

Our findings of declining gastrointestinal cancer risk over time, particularly colorectal cancer, are consistent with the results of some, but not all, studies from Denmark, Western Europe, and North America. Ekbom et al demonstrated a markedly increased risk of CRC in UC patients, based on follow-up data from 1922 to $1984 .{ }^{21}$ More recently two studies from Denmark have shown minimal or no increase in the relative risk of CRC in patients with $\mathrm{IBD}, 1,22$ during a period when the population rates have slightly increased ${ }^{23}$. However, a study in North America (where colorectal cancer rates have been falling ${ }^{23}$ ) failed to confirm this trend towards a lesser excess risk of colorectal cancer in patients with IBD. ${ }^{2}$ The declining excess risk of gastrointestinal malignancy among IBD patients in Denmark among may be due to earlier and more frequent use of immunosuppressive agents and biologics which may more effectively control inflammation, or other factors such as aminosalicylate use or more frequent colonoscopy and surgery in patients with UC.

Importantly, this study provides reassuring data that the declining risk for gastrointestinal malignancy observed here and in other recent Danish studies ${ }^{1,22}$ has not been offset by a concomitant increase in the risk of extra-intestinal or hematological malignancies. These findings suggest that changes in the medical and surgical treatment of IBD and/or other secular trends have not substantially impacted cancer relative risk on a population basis.

In regards to extra-intestinal malignancy, we found an increased risk of smoking-related malignancies in patients with $\mathrm{CD}$ but not $\mathrm{UC}$, consistent with prior literature. ${ }^{10}$ This is also consistent with the epidemiology of IBD, as smoking is a well-known risk factor for CD and protective factor for UC. As in prior studies, we have noted higher risks of melanoma in CD and NMSC in patients with both CD and UC. ${ }^{6-9}$ Taken together, these findings suggest preventive measures, including smoking cessation and routine skin examinations, may mitigate some of the excess cancer burden among IBD patients, particularly those with CD.

Despite suggestions from a few studies that female sexual hormones may increase the risk of $\mathrm{IBD}^{16,17}$, we found no association between IBD and female reproductive cancers. Our findings indicate an increased risk of hematological malignancies in patients with $\mathrm{CD}$, but not UC, a pattern that has been previously reported among IBD patients treated with immunosuppressive medications. ${ }^{3,5}$ Prior population-based studies and a recent metaanalysis did not demonstrate a significantly increased risk of lymphoma in the IBD population, though these prior studies were underpowered. ${ }^{10,24}$

Another noteworthy finding in this study is that relative risk of cancer in individuals diagnosed with IBD prior to 20 years of age is nearly twice that of individuals whose initial 
IBD diagnosis was recorded after 40 years of age. This is largely due to the risk of gastrointestinal cancers, in both $\mathrm{CD}$ and UC, as has been previously described. ${ }^{1}$ However, in $\mathrm{CD}$, the risk of extra-intestinal cancers also appears to be increased in those diagnosed with IBD at younger ages. We also found that patients with $\mathrm{CD}$ and $\mathrm{UC}$ were at higher risk of cancers occurring earlier in life, reinforcing the importance of initiating proper surveillance early in the course of disease.

The strengths of our study include its nationwide, population-based design, which ensures complete follow-up and virtually eliminates selection bias. Additionally, the large sample size and long study period spanning more than three decades allowed us to calculate distinct estimates of cancer risk for a number of different cancer types in patients with CD and UC, as well as to stratify these estimates by gender, age at IBD diagnosis, year of cancer diagnosis, age at cancer diagnosis, and time from IBD diagnosis. We used previously validated definitions of both the exposures ${ }^{14}$ and outcomes ${ }^{13}$ of interest, minimizing any misclassification.

Although our study was large, we did not have enough subjects to compute age-and sexspecific estimates of absolute cancer risks. The risks reported reflect an average over all subjects and sexes, and do not apply well to the youngest and oldest ages. Other limitations include the possibility of detection bias, i.e. detection of subclinical events more often in IBD patients than the general population due to more rigorous medical follow-up. This may partially explain the higher risk of gastrointestinal malignancy in the 1 year period following the first recorded IBD diagnosis, as well as less severe cancers such as NMSC. However, most of the cancers analyzed in this study would eventually have come to clinical attention and been included in the overall analysis, regardless of IBD status. Furthermore, the declining excess risk of gastrointestinal malignancy over time argues against significant detection bias. Another potential limitation is left censoring of the date of diagnosis of IBD. Individuals who presented clinically before 1978 or diagnosed as an outpatient before 1995 would have developed disease prior to the first recorded diagnosis. This would limit our ability to fully evaluate absolute risk as function of time of diagnosis and the association between age at IBD diagnosis and cancer risk. Finally, exclusion of patients with very mild disease and no inpatient encounters prior to 1995 may have led to an overestimation of gastrointestinal cancer risk, thereby accounting for some of the observed decreasing risk in later years. However, this extent of this bias should be minimal given that the Patient Registry included nearly all IBD patients (94\%) prior to the inclusion of outpatient visits in $1995 .^{14}$

In summary, patients with CD and UC are at increased risk of both intestinal and extraintestinal malignancies. The differing risk of specific cancers between CD and UC may reflect the underlying disease states, and/or lifestyle factors. The risk of gastrointestinal malignancy appears to be decreasing in recent years without a substantial increase in the overall risk of malignancy, which may suggest that the benefits of current management paradigms outweigh the cancer-related risks. 


\section{Supplementary Material}

Refer to Web version on PubMed Central for supplementary material.

\section{Acknowledgments}

Grant Support: This research was supported, in part, by National Institute for Diabetes and Digestive and Kidney Diseases Grant K08 DK088957-01 (MDK), National Institute for Diabetes and Digestive and Kidney Diseases Grant P30 DK034987 (RSS), and grants from the Karen Elise Jensen Foundation (HTS) and the Clinical Epidemiological Research Foundation (HTS).

\section{References}

1. Jess T, Simonsen J, Jorgensen KT, Pedersen BV, Nielsen NM, Frisch M. Decreasing risk of colorectal cancer in patients with inflammatory bowel disease over 30 years. Gastroenterology. 143:375-81 e1. quiz e13-4. [PubMed: 22522090]

2. Herrinton LJ, Liu L, Levin TR, Allison JE, Lewis JD, Velayos F. Incidence and mortality of colorectal adenocarcinoma in persons with inflammatory bowel disease from 1998 to 2010. Gastroenterology. 143:382-9. [PubMed: 22609382]

3. Beaugerie L, Brousse N, Bouvier AM, Colombel JF, Lemann M, Cosnes J, Hebuterne X, Cortot A, Bouhnik Y, Gendre JP, Simon T, Maynadie M, Hermine O, Faivre J, Carrat F. Lymphoproliferative disorders in patients receiving thiopurines for inflammatory bowel disease: a prospective observational cohort study. Lancet. 2009; 374:1617-25. [PubMed: 19837455]

4. Armstrong RG, West J, Card TR. Risk of cancer in inflammatory bowel disease treated with azathioprine: a UK population-based case-control study. Am J Gastroenterol. 2010; 105:1604-9. [PubMed: 20104215]

5. Kandiel A, Fraser AG, Korelitz BI, Brensinger C, Lewis JD. Increased risk of lymphoma among inflammatory bowel disease patients treated with azathioprine and 6-mercaptopurine. Gut. 2005; 54:1121-5. [PubMed: 16009685]

6. Long MD, Herfarth HH, Pipkin CA, Porter CQ, Sandler RS, Kappelman MD. Increased risk for non-melanoma skin cancer in patients with inflammatory bowel disease. Clin Gastroenterol Hepatol. 2010; 8:268-74. [PubMed: 20005977]

7. Peyrin-Biroulet L, Khosrotehrani K, Carrat F, Bouvier AM, Chevaux JB, Simon T, Carbonnel F, Colombel JF, Dupas JL, Godeberge P, Hugot JP, Lemann M, Nahon S, Sabate JM, Tucat G, Beaugerie L. Increased risk for nonmelanoma skin cancers in patients who receive thiopurines for inflammatory bowel disease. Gastroenterology. 141:1621-28. e1-5. [PubMed: 21708105]

8. Singh H, Nugent Z, Demers AA, Bernstein CN. Increased risk of nonmelanoma skin cancers among individuals with inflammatory bowel disease. Gastroenterology. 141:1612-20. [PubMed: 21806945]

9. Long MD, Martin CF, Pipkin CA, Herfarth HH, Sandler RS, Kappelman MD. Risk of melanoma and nonmelanoma skin cancer among patients with inflammatory bowel disease. Gastroenterology. 143:390-399 e1. [PubMed: 22584081]

10. Pedersen N, Duricova D, Elkjaer M, Gamborg M, Munkholm P, Jess T. Risk of extra-intestinal cancer in inflammatory bowel disease: meta-analysis of population-based cohort studies. Am J Gastroenterol. 2010; 105:1480-7. [PubMed: 20332773]

11. Frank L. Epidemiology. When an entire country is a cohort. Science. 2000; 287:2398-9. [PubMed: 10766613]

12. Lynge E, Sandegaard JL, Rebolj M. The Danish National Patient Register. Scand J Public Health. 39:30-3. [PubMed: 21775347]

13. Storm HH, Michelsen EV, Clemmensen IH, Pihl J. The Danish Cancer Registry-history, content, quality and use. Dan Med Bull. 1997; 44:535-9. [PubMed: 9408738]

14. Fonager K, Sorensen HT, Rasmussen SN, Moller-Petersen J, Vyberg M. Assessment of the diagnoses of Crohn's disease and ulcerative colitis in a Danish hospital information system. Scand J Gastroenterol. 1996; 31:154-9. [PubMed: 8658038] 
15. Andersen TF, Madsen M, Jorgensen J, Mellemkjoer L, Olsen JH. The Danish National Hospital Register. A valuable source of data for modern health sciences. Dan Med Bull. 1999; 46:263-8. [PubMed: 10421985]

16. Jess T, Frisch M, Jorgensen KT, Pedersen BV, Nielsen NM. Increased risk of inflammatory bowel disease in women with endometriosis: a nationwide Danish cohort study. Gut. 61:1279-83. [PubMed: 22184069]

17. Garcia Rodriguez LA, Gonzalez-Perez A, Johansson S, Wallander MA. Risk factors for inflammatory bowel disease in the general population. Aliment Pharmacol Ther. 2005; 22:309-15. [PubMed: 16097997]

18. Mahid SS, Minor KS, Soto RE, Hornung CA, Galandiuk S. Smoking and inflammatory bowel disease: a meta-analysis. Mayo Clin Proc. 2006; 81:1462-71. [PubMed: 17120402]

19. Jemal A, Siegel R, Xu J, Ward E. Cancer statistics, 2010. CA Cancer J Clin. 60:277-300. [PubMed: 20610543]

20. Rothman, KJ.; BJ. Epidemiologic analyses with a programmable calculator. Washington, D.C.: Government Printing Office; 1979. DHHS publication no. (NIH) 79-1649.)

21. Ekbom A, Helmick C, Zack M, Adami HO. Ulcerative colitis and colorectal cancer. A populationbased study. N Engl J Med. 1990; 323:1228-33. [PubMed: 2215606]

22. Winther KV, Jess T, Langholz E, Munkholm P, Binder V. Long-term risk of cancer in ulcerative colitis: a population-based cohort study from Copenhagen County. Clin Gastroenterol Hepatol. 2004; 2:1088-95. [PubMed: 15625654]

23. Center MM, Jemal A, Ward E. International trends in colorectal cancer incidence rates. Cancer Epidemiol Biomarkers Prev. 2009; 18:1688-94. [PubMed: 19505900]

24. Loftus EV Jr, Tremaine WJ, Habermann TM, Harmsen WS, Zinsmeister AR, Sandborn WJ. Risk of lymphoma in inflammatory bowel disease. Am J Gastroenterol. 2000; 95:2308-12. [PubMed: 11007233] 


\section{Crohn's disease}

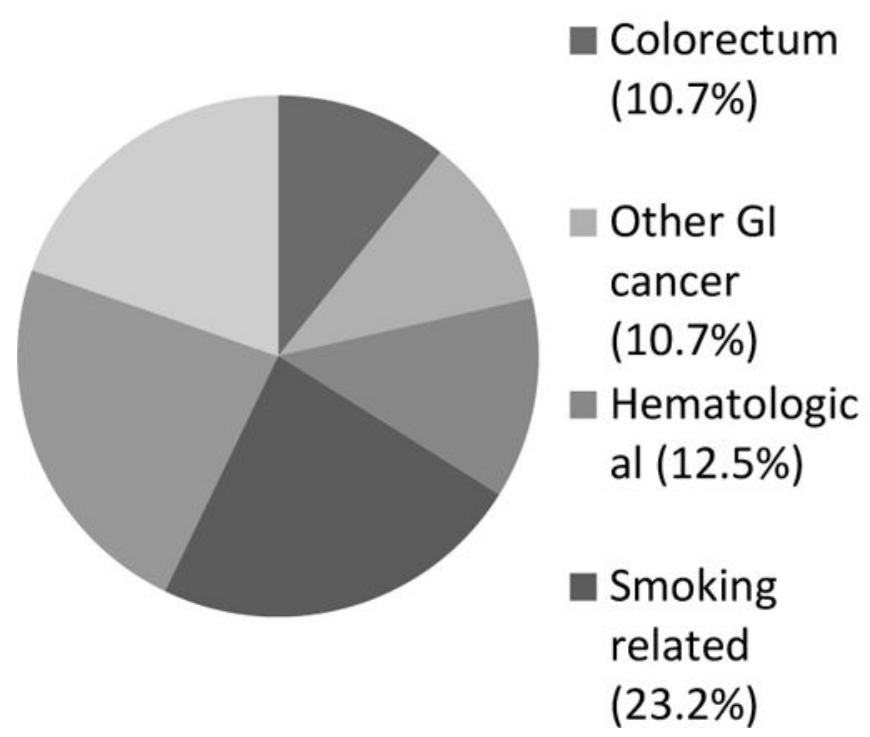

\section{Ulcerative Colitis}

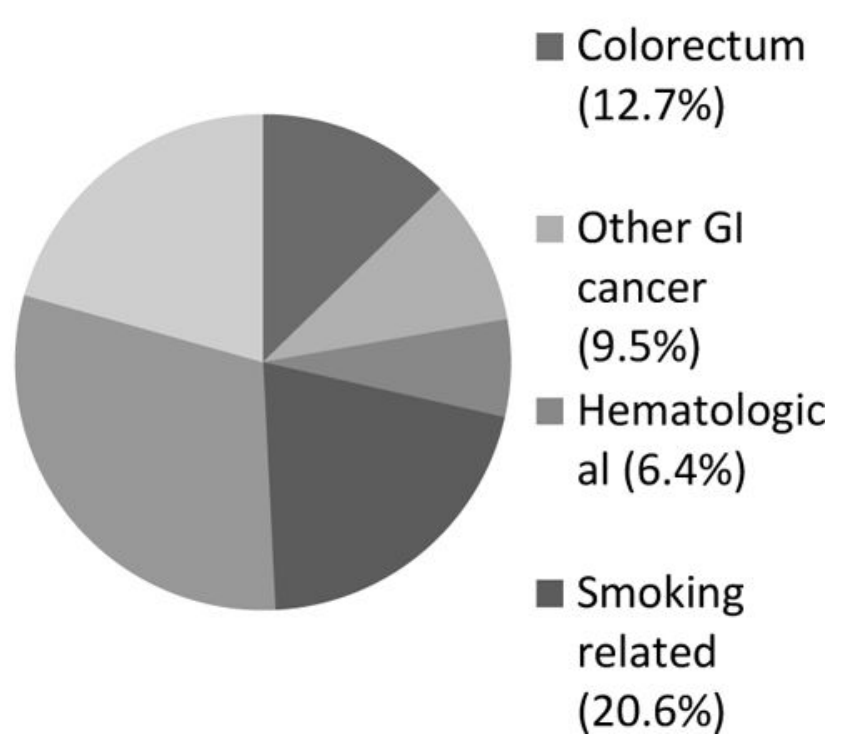

Figure 1.

Absolute risk of invasive malignancy 1-11 years following IBD diagnosis, Denmark 19782010. 


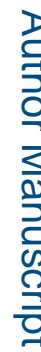

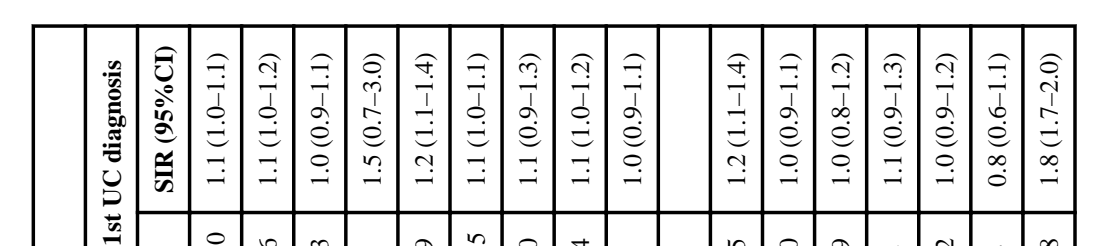

ב্

ב

롬ำ

ิิ

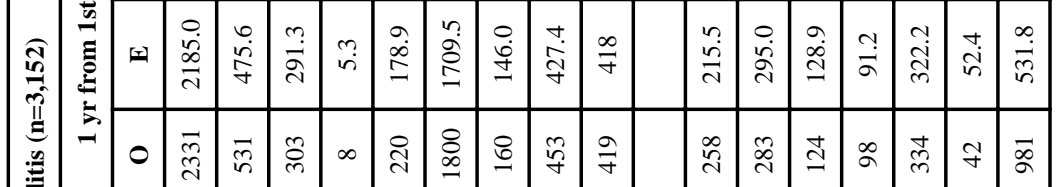

苗



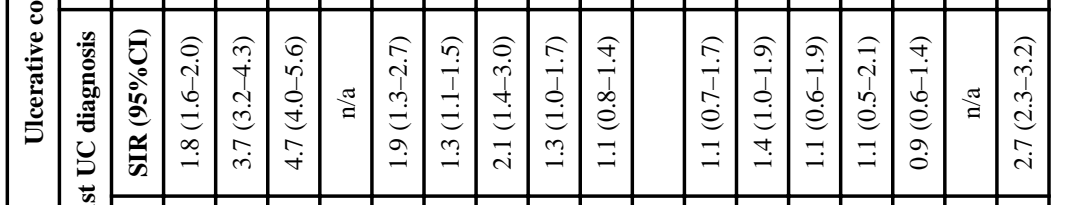

로을

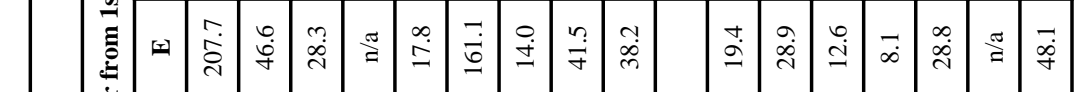

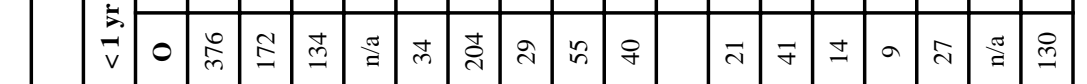$$
3
$$

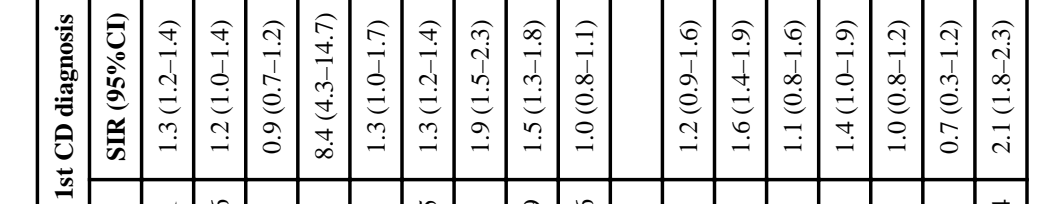

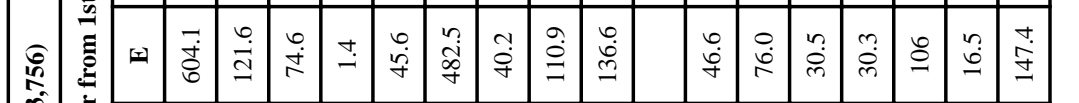

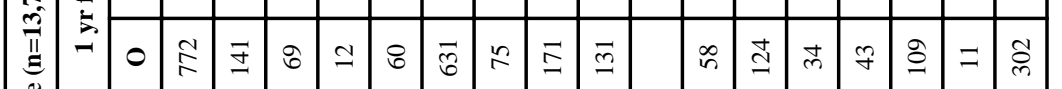

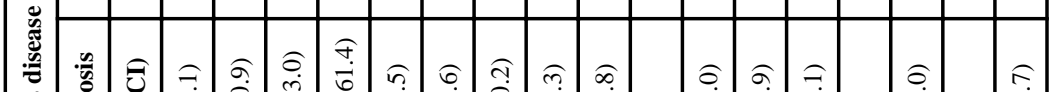

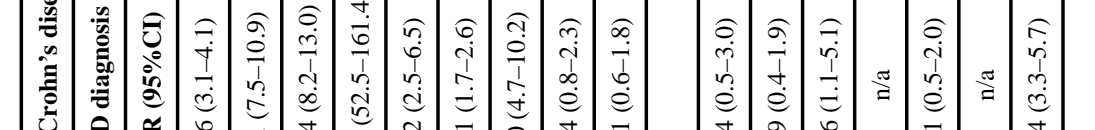

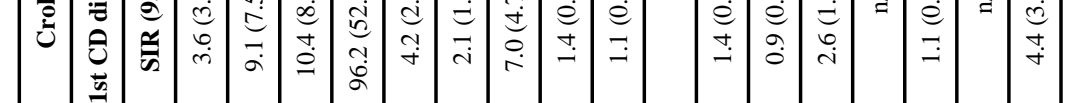

ثี

華

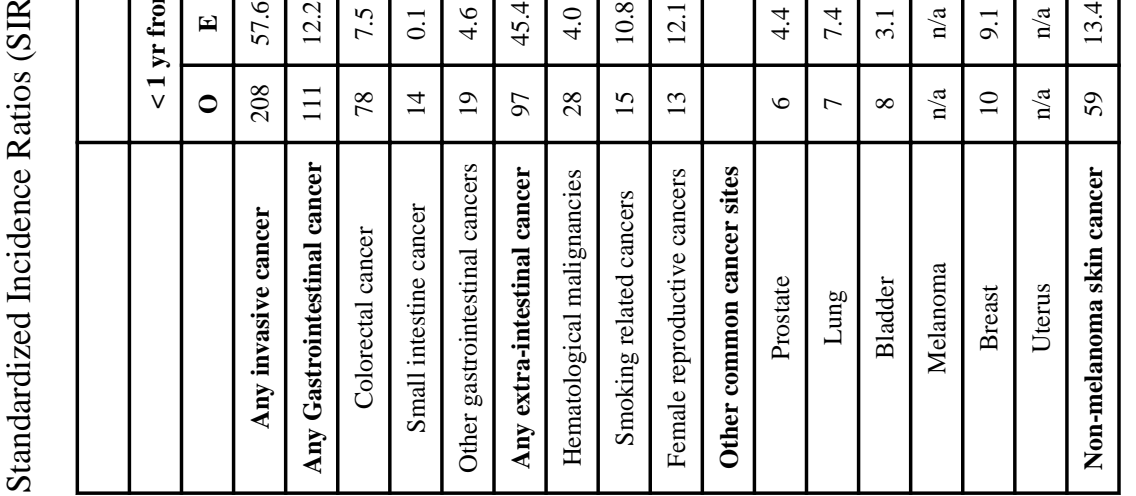

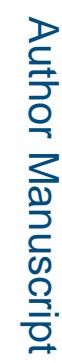

Clin Gastroenterol Hepatol. Author manuscript; available in PMC 2015 March 17. 
Table 2

Absolute Risk of Selected Cancers occurring 1-11 years following a diagnosis of Inflammatory Bowel Diseases (IBD), Denmark, 1978-2010

\begin{tabular}{|c|c|c|}
\hline & $\begin{array}{c}\text { Crohn's disease (n=13,756) } \\
\text { Absolute risk percent (95\% CI) }\end{array}$ & $\begin{array}{c}\text { Ulcerative colitis (n=35,152) } \\
\text { Absolute risk percent (95\% CI) }\end{array}$ \\
\hline Any invasive cancer & $5.6(5.1-6.1)$ & $6.3(6.0-6.6)$ \\
\hline Any gastrointestinal cancer & $1.2((1.0-1.4)$ & $1.4(1.2-1.6)$ \\
\hline Colorectal cancer & $0.6(0.4-0.8)$ & $0.8(0.7-0.9)$ \\
\hline Small intestine cancer & $0.07(0.03-0.15)$ & $0.03(0.01-0.06)$ \\
\hline Other GI cancers & $0.5(0.4-0.7)$ & $0.6(0.5-0.7)$ \\
\hline Any extra-intestinal cancer & $4.4(4.0-4.9)$ & $5.0(4.7-5.3)$ \\
\hline Hematological malignancies & $0.7(0.5-0.9)$ & $0.4(0.3-0.5)$ \\
\hline Smoking related cancers & $1.3(1.1-1.5)$ & $1.3(1.2-1.5)$ \\
\hline Female reproductive cancers & $0.9(0.7-1.1)$ & $12(1.1-1.3)$ \\
\hline Other common cancer sites & & $0.7(0.6-0.8)$ \\
\hline Prostate & $0.4(0.3-0.5)$ & $0.8(0.7-1.0)$ \\
\hline Lung & $0.9(0.7-1.1)$ & $0.4(0.3-0.4)$ \\
\hline Bladder & $0.2(0.1-0.3)$ & $0.3(0.2-0.4)$ \\
\hline Melanoma & $0.3(0.2-0.5)$ & $1.0(0.8-1.1)$ \\
\hline Breast & $0.8(0.6-1.0)$ & $0.1(0.1-0.2)$ \\
\hline Uterus & $0.07(0.03-0.14)$ & $3.1(2.9-3.3)$ \\
\hline Non-melanoma skin cancer & $2.2(1.9-2.5)$ & \\
\hline & & \\
\hline
\end{tabular}




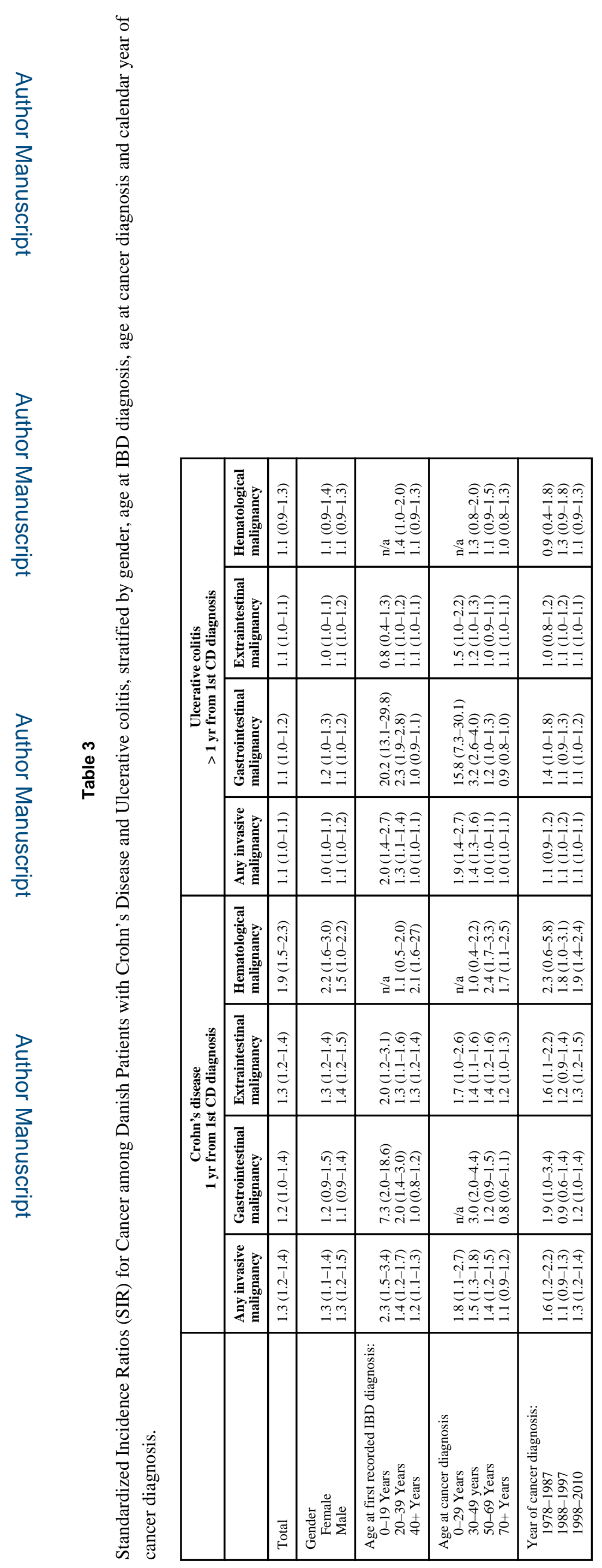

